\title{
Bidirectional modification of gears in the generalized theory of meshing
}

\author{
Ognyan Alipiiev \\ Department of Theory of Mechanisms and Machines, University of Ruse, Ruse 7017, Bulgaria
}

\begin{abstract}
An important part of "Generalized theory", developed by the author of this article, is the "bidirectional modification" of involute gears. The main point of the proposed modification is that together with the traditional "radial modification" of the basic rack, an additional "tangential modification" of the side profiles of the rack-cutters is introduced. As a result, the area of possible existence of involute gear drives in the generalized theory is considerably extended. Based on the bidirectional modification, a new classification of involute gears at their meshing with a rack cutter is proposed. The classification is characterized by a significant variety, which is composed of thirteen different types of gears: a zero gear; two radially modified gears; two tangentially modified gears and eight two-way modified gears. Based on the bidirectional modification in radial and tangential directions, a new classification of the involute gear drives is proposed. The classification is composed of sixteen different types of gear drives: a zero gear drive, three equally-modified gear drives; two balanced gear drives; five positive gear drives and five negative gear drives.
\end{abstract}

\section{Introduction}

Nowadays, the traditional theory (TT) [1-4] for geometric design of involute gear drives, developed in the course of many years, is the most widespread. In this theory, the traditional basic rack (TBR) is a fundamental principle. Essentially, TT is directly related to gear production and TBR is used to determine the geometric form of the gears and the gear-cutting tool. A main principle in TT is the direct dependency of the gear drives geometry on the rack-cutter geometry (gearcutting tool) used for cutting the gear teeth. Hence in TT, after the independent parameters of TBR are set, the rack-cutter geometric form is defined first. After that, the geometry of both gears from the gear drive is found in the instrumental meshing with the same rack-cutter. Finally, the geometry of the gear meshing is defined. Furthermore, the geometry of TBR is defined unambiguously by four independent parameters and the geometry of the gear drive by eight independent parameters.

Despite its undeniable advantages, the TT has one essential disadvantage which consists of a comparatively narrow area of involute meshing existence, determined by this theory. This disadvantage becomes clearer in the geometric design of gear drives with a small number of teeth and a greater contact ratio.

To extend the existence area of the involute meshing, the Russian scientist E. B. Vulgakov [5] developed another non-traditional theory which has been popularized by his follower A. L. Kapelevich under the name "Direct Gear Design" (DGD) [6]. The main approach in developing DGD is excluding the TBR as a fundamental principle in involute geometry design and introducing new independent parameters, directly connected to the involute profile. Applying such an approach in which the geometry of the meshed gears is not previously bound with the gear cutting tool significantly extends the design possibilities. However, the positive results obtained have not contributed to mass application of DGD in production, mainly because of its unpopular principles. Furthermore, rather than developing the popular TT, DGD denies it.

An increased interest in involute drives, formed by gears with asymmetric teeth profiles [7-11], has been observed lately. These gear drives can be successfully applied in automotive transmissions, wind turbines, gear pumps, cargo lifting mechanisms, etc. The asymmetric meshing is appropriate when the main load in the gear drive is transmitted primarily one-way or when the drive is irreversible. The application of asymmetric teeth has some advantages, and the most important one is increasing the load capacity in the active direction of the gear drive. This advantage is achieved by increasing the pressure angle of the drive side profiles and decreasing it in the coast side ones $[12,13]$.

To overcome these disadvantages of TT and DGD and for summary presentation of the geometric theory of symmetric and asymmetric meshing, the author proposes a generalized model for geometric design of cylindrical gear drives with symmetric, asymmetric, spur and helical teeth. The proposed generalized model of meshing allows a generalized theory (GT) for geometric design of involute gear drives with both symmetric and asymmetric teeth [14-18, 21], to be developed. It unites the advantages of TT and DGD and its methodology is based on developing and improvement of TT, instead of its denial. 
The three fundamental parts in the proposed GT are the asymmetric meshing, the generalized basic rack and the bidirectional modification gears. The so-called generalized involute meshing is placed the highest in the GT hierarchy. Essentially, it is asymmetric meshing defined by 20 independent parameters. Furthermore, a new generalized basic rack (GBR) [19] is determined as a fundamental principle in GT. By GBR the geometry of involute meshing is presented in the most generalized way. Unlike TBR, the proposed GBR has asymmetric profiles and it is defined by twelve independent parameters, instead of four. In GT together with the traditional radial modification, the so-called tangential modification is also introduced, in which the gears are modified in both direction at the same time. Combining the radial modification with tangential, i.e. introducing bidirectional modification, significantly increases the variations of gears. Besides, the variations of involute meshing are increased, whereby the area of their application is expanded. Furthermore, the strength of the gear drive is increased and the sharpening of the teeth is avoided by the bidirectional modification [20].

This article is dedicated to the third main part of GT, related to bidirectional modification of involute gears. A new classification of gears and gear drives is proposed based on the bidirectional modification. It includes many more varieties than the known traditional types.

\section{Essence of the bidirectional modification}

It becomes clear from GT that from all possible cases, the most general is the one shown in Fig.1, in which the gear is bidirectionally modified (radially and tangentially) and, at the same time, the side teeth profiles are asymmetric. In this general case the relative location of the rack cutter (the rack-tool) against the gear it is cutting is developed by shifting the rack-cutter profiles in two directions - radial and tangential. In the traditional theory, as well as in GT, the term, "shift of the basic rack" (shift of the GBR) is accepted instead of "shift of the instrumental teeth profiles". Also, the gears are called, "modified" when the respective shift of GBR is realized.

The values of the radial modification $X$ and the tangential modification $X_{\tau}$ are defined by the equations

$$
X=x m, \quad X_{\tau}=x_{\tau} m,
$$

in which $m$ is the module, and $x$ and $x_{\tau}$ are the radial and the tangential modification coefficients of GBR, respectively. These coefficients are dimensionless and the module dimension is in millimeters, and as a result, the shifts $X$ and $X_{\tau}$ of GBR are measured also in millimeters.

When the gears are molded, all of these coefficients $x$ and $x_{\tau}$ can have positive, negative or zero value. In

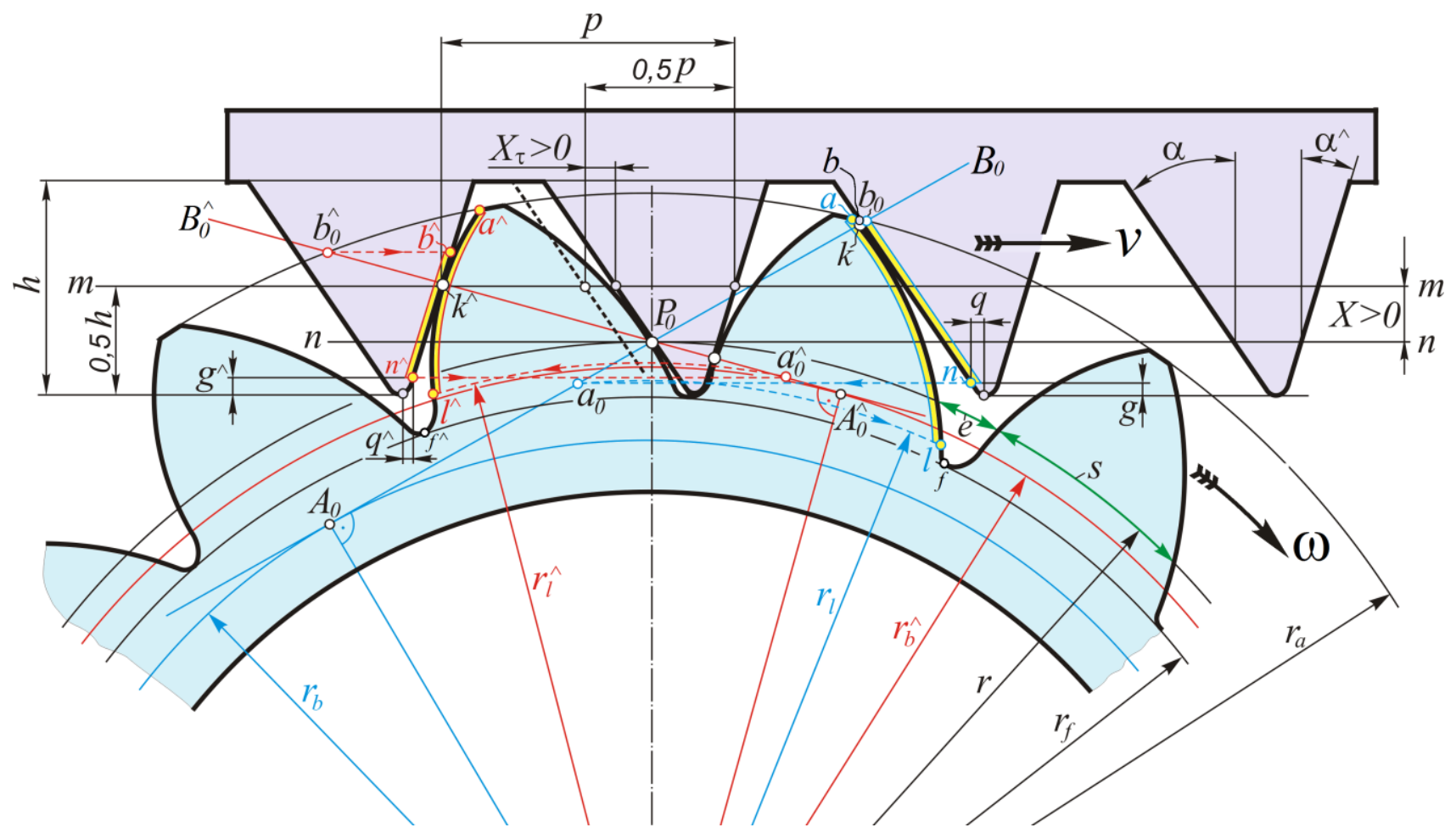

Fig.1. Generalized meshing rack-cutter tools with gear: $z=16 ; x=0.6 ; x_{\tau}=0.3$.

Fig. 1 is shown the case when the gear has 16 teeth and it is positively modified in both radial and tangential directions $\left(x=0.6, x_{\tau}=0.3\right)$. From this figure it is clear that the positive values of $x$ and $x_{\tau}$ lead to increasing the thickness of the teeth $s$ and decreasing the width $e$ of the inter teeth on the gear reference circle $r$. The thickness of the teeth $s$ in GT is defined by equation [15]

$$
s=m\left[\pi / 2+x_{\tau}+x\left(\tan \alpha+\tan \alpha^{\wedge}\right)\right],
$$


in which it is clear that at negative values of $x$ and $x_{\tau}$ this thickness $s$ decreases.

Defining the types of respective modification of gears depends directly on the width of the rack-cutter inter teeth on the reference straight line $m-m$ and the pitch straight line $n-n$. In the meshing rack-cutter tools (Fig.1) the pitch straight line $n-n$ touches the reference circle with radius $r$ and the reference straight line $m-m$ divides the height of the rack teeth on two equal parts.

When the radial and tangential shift coefficients have zero values at the same time $\left(x=0, x_{\tau}=0\right)$, the reference straight line $m-m$ coincides with the pitch straight line $n-n$. Then both the tooth thickness $s$ and the width of the inter tooth $e$ on the gear reference circle, as well the thickness of the tooth and the width of the inter tooth at the reference line and the pitch line of the rack-cutter, have equal values, defined by equation

$$
s=e=\frac{\pi m}{2} .
$$

At $x_{\tau}=0$, the tooth thickness and the inter tooth on the reference line $m-m$ of the rack-cutter are equal. If in this case the coefficient $x$ has value $x \neq 0$, the width of the inter tooth and the thickness of the tooth on the pitch line $n-n$ change, as a result

$$
s \neq e \neq \frac{\pi m}{2} .
$$

The requirement (4) applies also at $x_{\tau} \neq 0, x=0$ and when $x_{\tau} \neq 0, x \neq 0$

In the realization of the bidirectional modification of the gears, according to the signs and values of $x$ and $x_{\tau}$, a multitude of different varieties is obtained. They can be generally grouped in four typical cases:

$1^{0}$ Zero gear in which $x=0, x_{\tau}=0$ and $s=e$;

$2^{0}$ Positive gear, in which due to modification $s>e$;

$3^{0}$ Negative gear, in which due to modification $s<e$;

$4^{0}$ Balance gear, in which $x \neq 0, x_{\tau} \neq 0$ and $s=e$.

When a bidirectional modification is realized and the gear is positive (the second case) it is possible for one of the coefficients $x$ or $x_{\tau}$ to have a negative value, but due to the predominant positive value of the other $s>e$ may be obtained. Similarly, at negative gear (the third case), it is possible one coefficient $x$ or $x_{\tau}$ to have a negative value and, at the same time, the requirement $s<e$ to be fulfilled. The fourth case, in which the gear is balanced, is specific only for the newly proposed theory, i.e. at bidirectional modification of the gears. In this case the coefficients $x$ or $x_{\tau}$ have different signs but the relation between them is such that the requirement $s=e$ is fulfilled. Then from equation (2) it is clear that if the requirement (5) is fulfilled

$$
x_{\tau}+x\left(\tan \alpha+\tan \alpha^{\wedge}\right)=0,
$$

in different values of $x$ and $x_{\tau}$ the thickness of the tooth $s$ is equal to the width of the inter tooth $e$, i.e. $s=e=p / 2=\pi m / 2$.

\section{Classification of gears}

According to the classification proposed in Fig. 2, involute gears with symmetric and asymmetric teeth can be grouped in general as zero (unmodified) and modified.

The zero gears are marked with $\mathbf{Z 0}$ and in the modified gears sign the symbol $\mathbf{M}$ is included ( $M$ is the first letter of the term „,modification”). Further, the gear is zero when the radial and tangential coefficients are $x=0$ and $x_{\tau}=0$. If one (or both) coefficients are different from zero, the gear is modified.

The modified gears are also grouped into:

1) Radially modified - MR, when $x \neq 0$ and $x_{\tau}=0$;

2) Tangentially modified - MT , when $x=0$ and $x_{\tau} \neq 0$

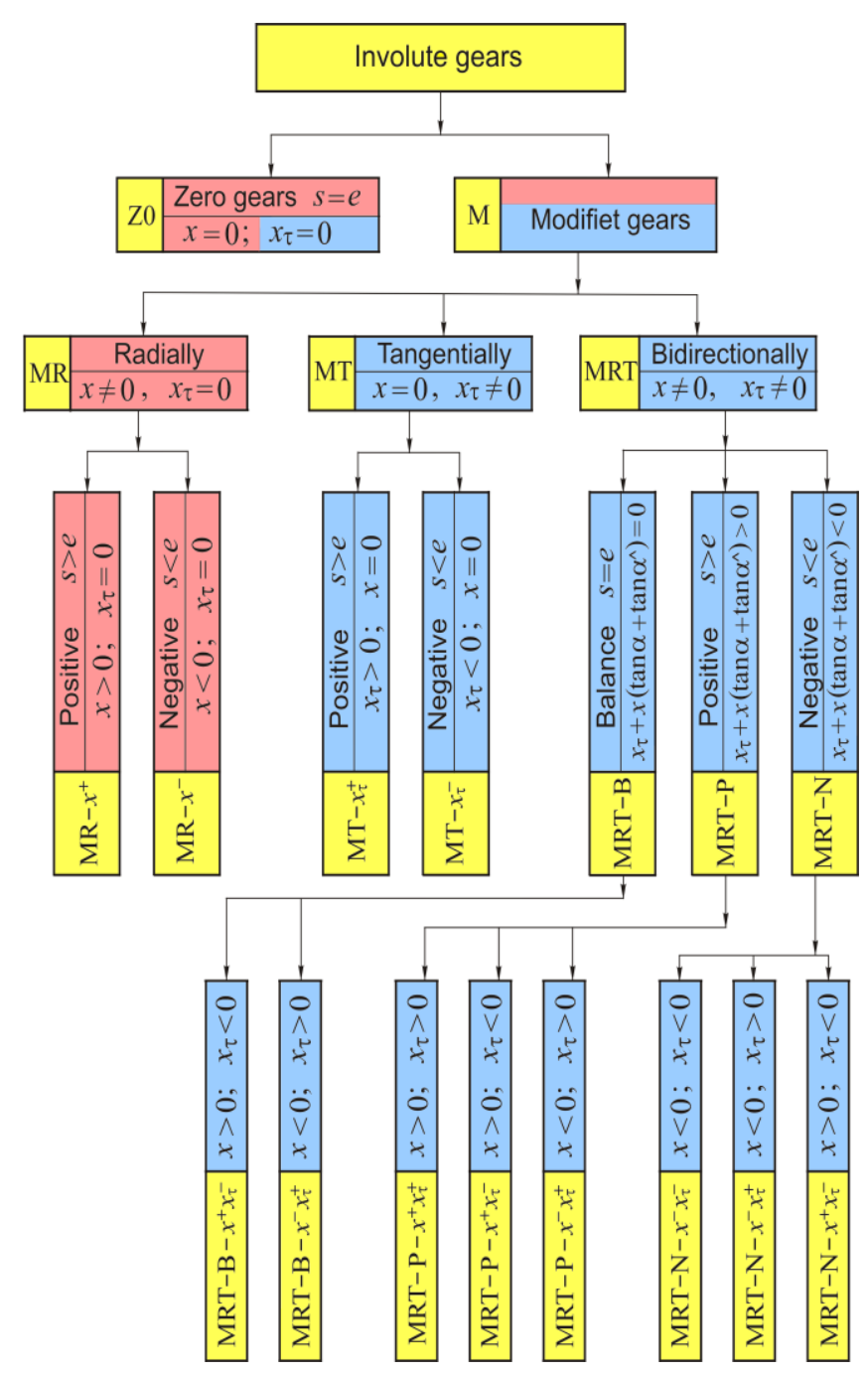

Fig.2. Gear classification. 
3) Bidirectionally modified - MRT (Modified Radially and Tangentially), when $x \neq 0$ and $x_{\tau} \neq 0$.

Depending on the respective sign (plus or minus) of the shift coefficient, the radially modified gears are two types:

1a) Positive - MR- $x^{+}$, when $x>0$ and $x_{\tau}=0$;

$1_{\mathrm{b}}$ ) Negative - MR- $\boldsymbol{x}^{-}$, when $x<0$ and $x_{\tau}=0$.

Similarly, tangentially modified gears are also two types:

2a) Positive - MT- $\boldsymbol{x}_{\tau}^{+}$, when $x=0$ and $x_{\tau}>0$;

$2 \mathrm{~b}$ ) Negative - MT- $\boldsymbol{x}_{\tau}^{-}$, when $x=0$ and $x_{\tau}<0$.

When the modification is radial and tangential at the same time, the varieties of the gears are much more. In this case, the distinguishing feature is the values of $s$ and $e$. Then three types of bidirectional modification are possible:

$3_{\mathrm{a}}$ ) Balance - MRT-B , $s=e$;

$3_{\mathrm{b}}$ ) Positive - MRT-P, $s>e$;

$3_{\mathrm{c}}$ ) Negative - MRT-N, $s<e$.

Moreover, according to the accepted signs (plus or minus) of the shift coefficients $x$ and $x_{\tau}$ eight different types are possible.

In Fig. 3 is shown an asymmetric meshing rack-cutter tools and zero gear Z0 with respective shifts: $X=0$ and $X_{\tau}=0$. In this case the reference line $m-m$ from the rack-cutter coincides with the pitch line $n-n$, and as a result $s=e$.

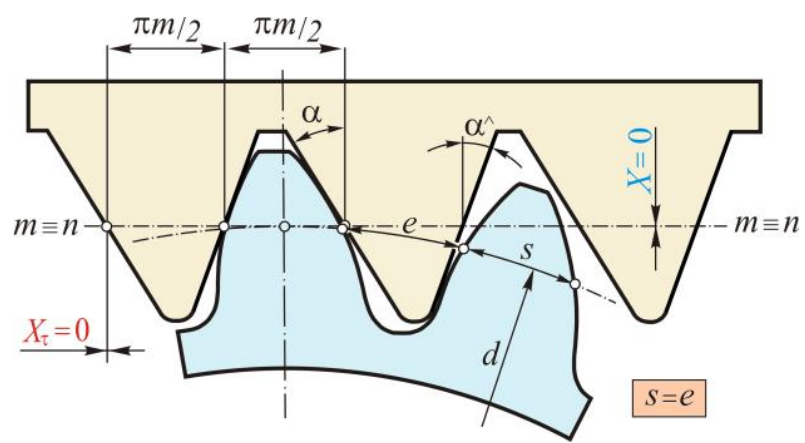

Fig. 3. Zero gear-Z0: $x=0 ; x_{\tau}=0$.

When the gears are radially modified (Fig. 4) the pitch and reference lines from the rack-cutter coincide, because of which $s \neq e$. If the radial modification is positive $(X>0)$, the rack-cutter is moved away from the gear center, and at $X<0-$ the rack is moved closer. Due to this shift at positive gear (MR- $\left.x^{+}\right) s>e$ (Fig. 4a), and at negative gear (MR- $\left.x^{-}\right) s<e$ (Fig. 4b).

The meshing shown in Fig. 5 is between gears modified only tangentially $\left(X=0, X_{\tau} \neq 0\right)$. In this case the straight lines $n-n$ and $m-m$ coincide with each other because of tangential modification $s \neq e$. Moreover, $s>e$ if $X_{\tau}>0$ (gear MT- $x_{\tau}{ }^{+}$in Fig. 5a) and $s<e$ at $X_{\tau}<0$ (gear MT- $x_{\tau}^{-}$in Fig. 5 b).
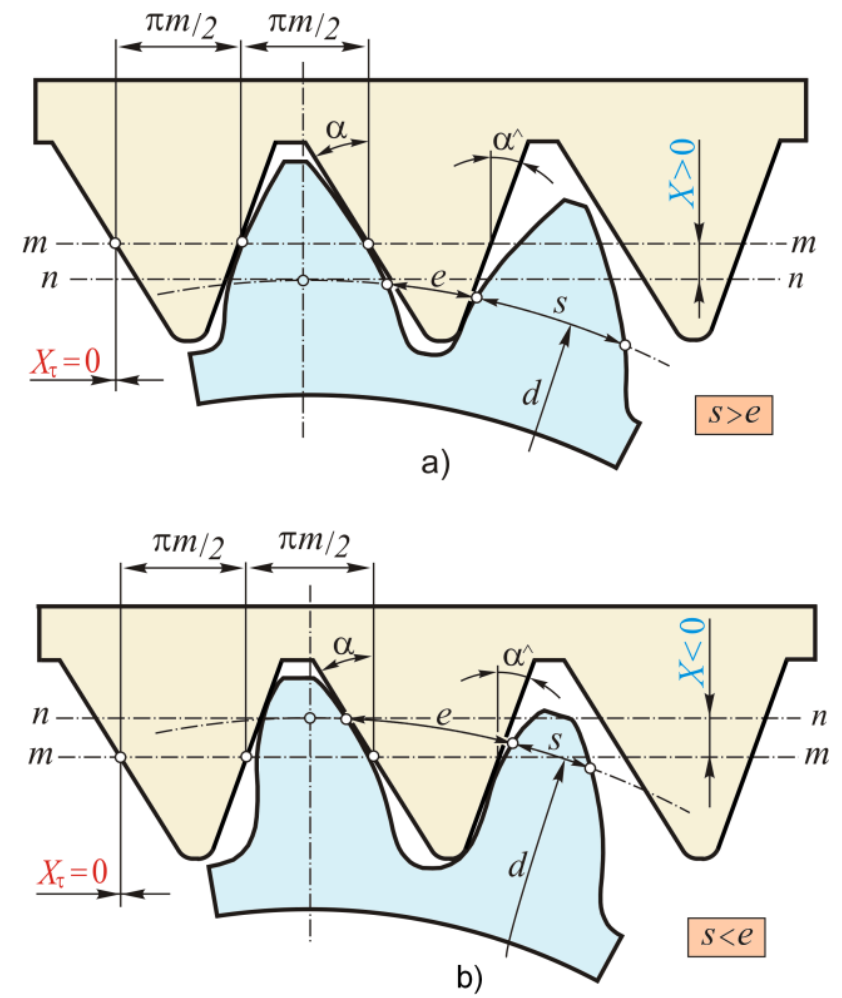

Fig. 4. Radially modified gears:

a) MR- $\boldsymbol{x}^{+}$(positive $x>0 ; x_{\tau}=0$ ),

b) MR- $\boldsymbol{x}^{-}$(negative $\left.x<0 ; x_{\tau}=0\right)$.

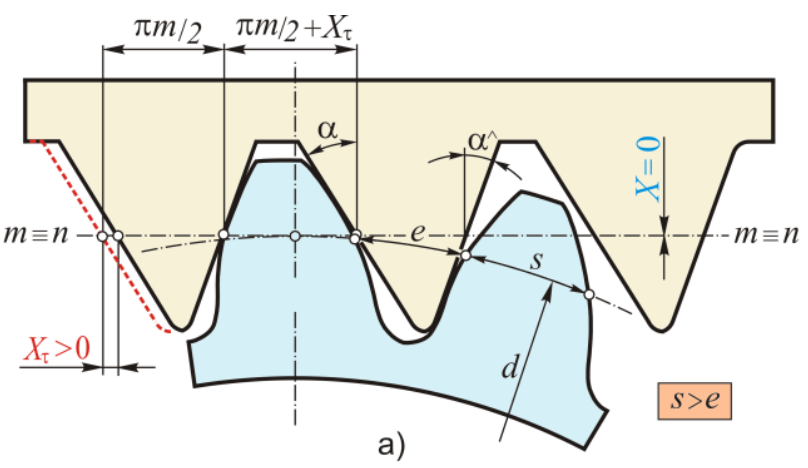

a)

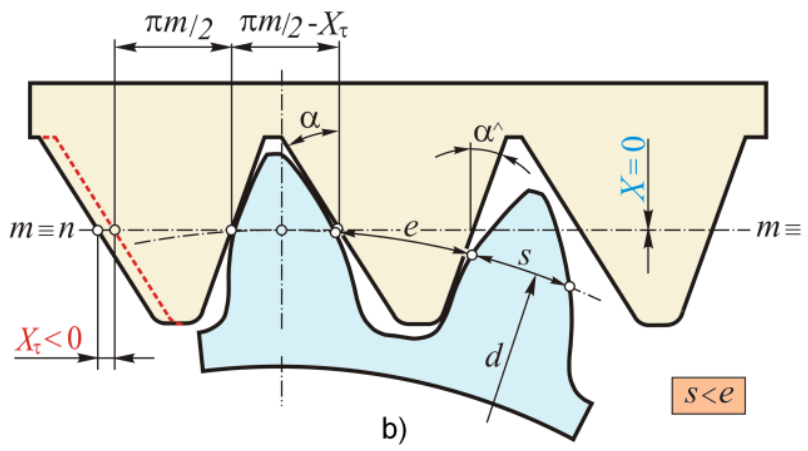

Fig. 5. Tangentially modified gears:

a) MT- $\boldsymbol{x}^{+}$(positive $\left.x=0 ; x_{\tau}>0\right)$,

b) MT- $\boldsymbol{x}^{-}$(negative $x=0 ; x_{\tau}<0$ ). 


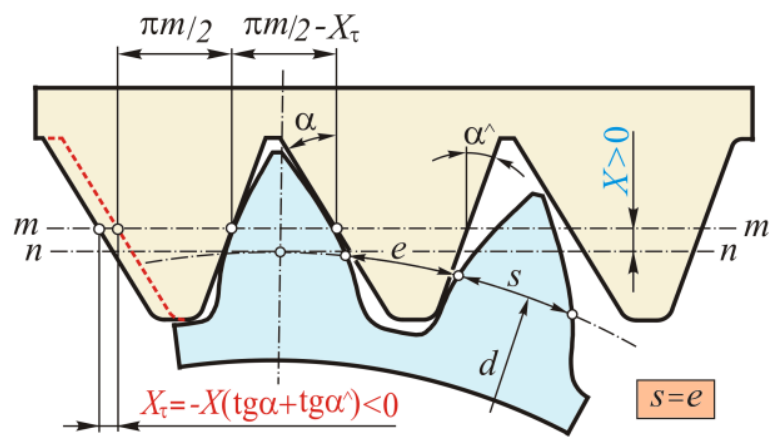

a)

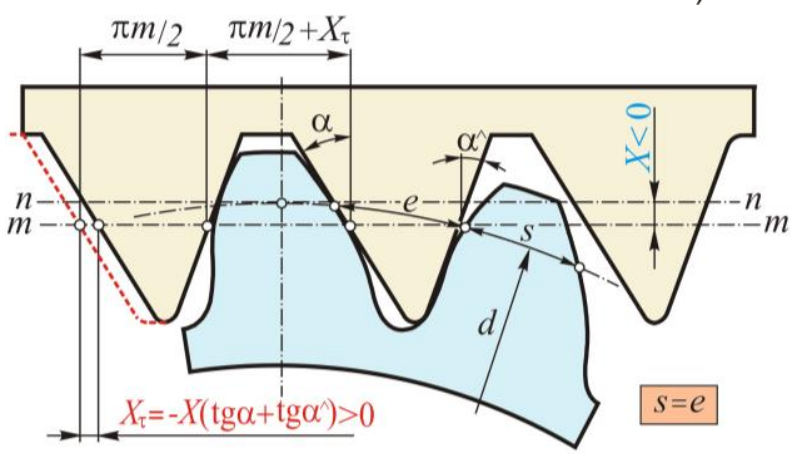

b)
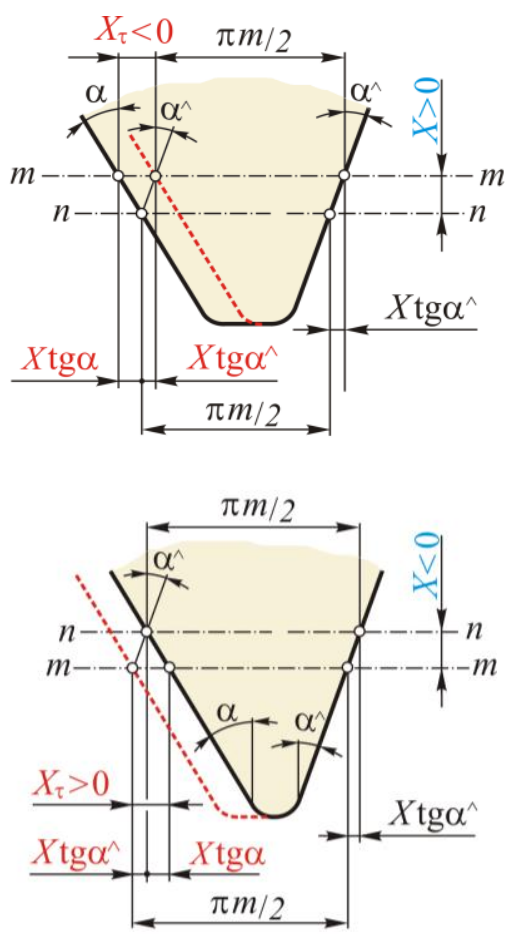

Fig. 6. Bidirectionally modified balance gears:

a) MRT-B $-\boldsymbol{x}^{+} \boldsymbol{x}_{\tau}^{-}\left(x>0 ; x_{\tau}<0\right)$, b) MRT-B - ${ }^{-} \boldsymbol{x}_{\tau}^{+}\left(x=<0 ; x_{\tau}>0\right)$.
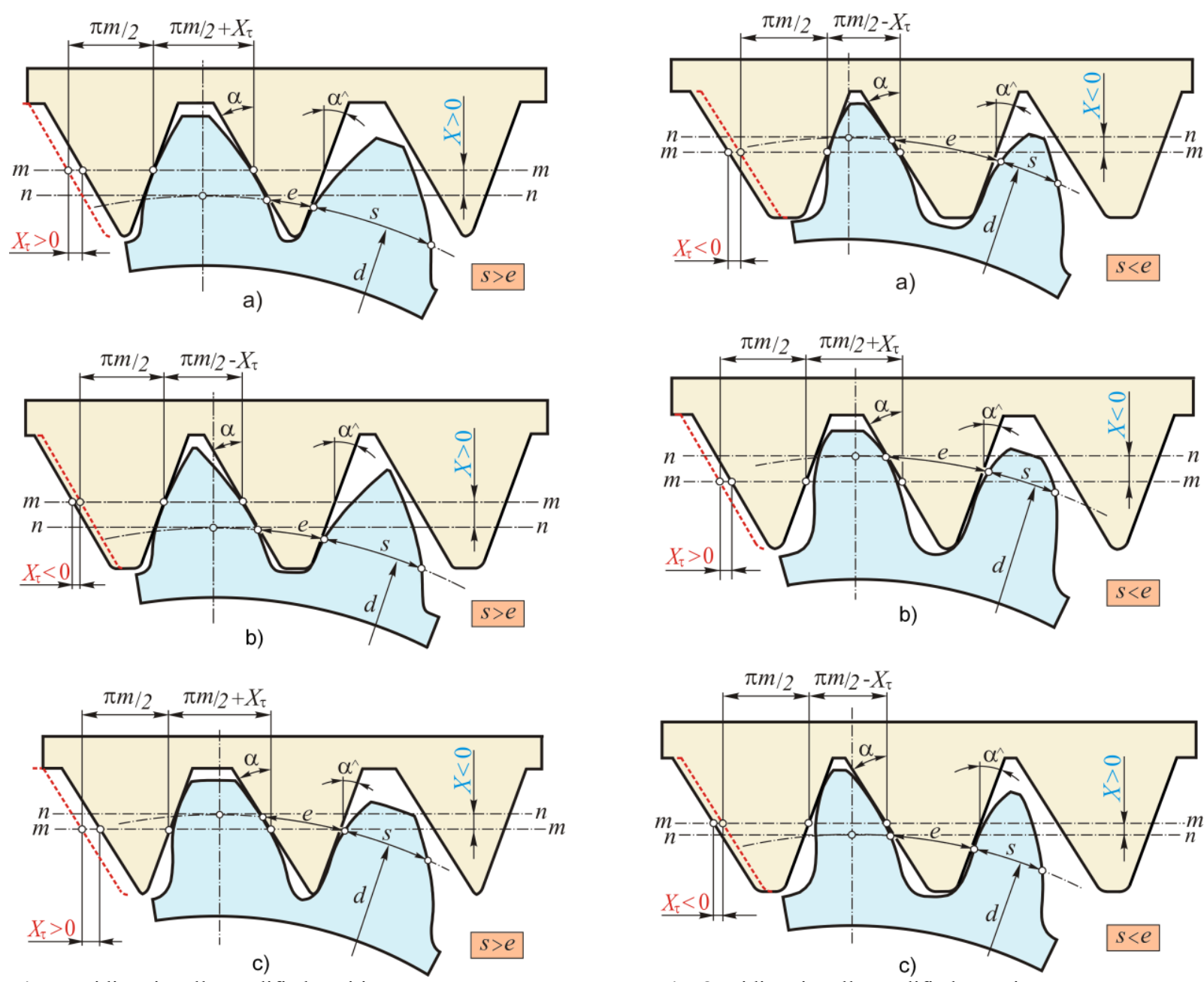

Fig. 7. Bidirectionally modified positive gears:

a) MRT-P - $\boldsymbol{x}^{+} \boldsymbol{x}_{\tau}^{+}$, b) MRT-P - $\boldsymbol{x}^{+} \boldsymbol{x}_{\tau}^{-}$, c) MRT-P - $\boldsymbol{x}^{-} \boldsymbol{x}_{\tau}^{+}$.

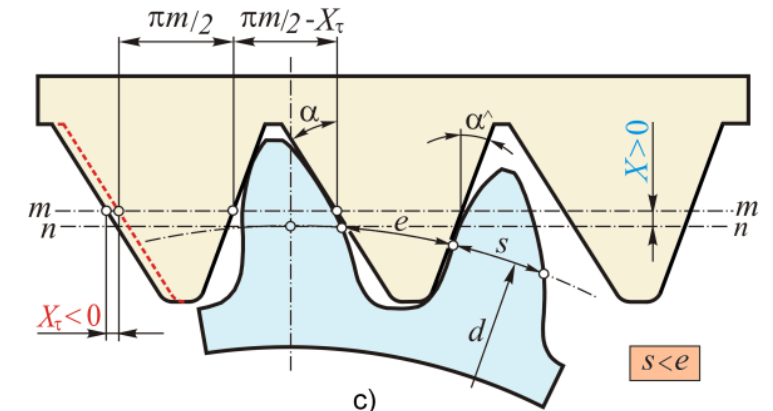

Fig. 8. Bidirectionally modified negative gears:

a) MRT-N $-\boldsymbol{x}^{-} \boldsymbol{x}_{\tau}^{-}$, b) MRT-N $-\boldsymbol{x}^{-} \boldsymbol{x}_{\boldsymbol{\tau}}^{+}$, c) MRT-N $-\boldsymbol{x}^{+} \boldsymbol{x}_{\tau}^{-}$ 
The number of instrumental meshings increases significantly when the gears are bidirectionally modified (Fig. 6, Fig. 7, Fig. 8).

At bidirectional balance modification the straight lines $n-n$ and $m-m$ do not coincide with each other (Fig. 6) but there is a definite relation $X_{\tau}=-X\left(\tan \alpha+\tan \alpha^{\wedge}\right)$ between the shifts $X$ and $X_{\tau}$, by which the equation $s=e$ is fulfilled. Furthermore, according to the signs of $X$ and $X_{\tau}$, two different varieties are possible. In the first case (Fig. 6a) the balance gear MRT-B $-x^{+} x_{\tau}^{-}$is characterized with positive sign for radial shift and negative sign for tangential shift. In the second case (Fig. 6b) the signs of radial and tangential shifts for the balance gear MRT-B $x^{-} x_{\tau}^{+}$are opposite of the first case $\left(X>0\right.$ and $\left.X_{\tau}>0\right)$.

When the gears are bidirectionally modified, the requirement $s>e$ (Fig. 7) is fulfilled. These gears are generally positively modified despite the fact that the radial or tangential shift may have negative value. In this case, three varieties are possible: gear MRT-P $-x^{+} x_{\tau}^{+}$ (Fig. 7a) in which the two shifts are positive $(X>0$, $X_{\tau}>0$ ); gear MRT-P $-x^{+} x_{\tau}^{-}$(Fig. 7b) when $X>0$, $X_{\tau}<0$; gear MRT-P $-x^{-} x_{\tau}^{+}$(Fig. 4c) in which $X<0$, $X_{\tau}>0$.

The bidirectionally modified gears are generally negatively modified (Fig. 8) when the inequality $s<e$ is fulfilled. Here three varieties are possible, too: gear MRT-N $-x^{-} x_{\tau}^{-}$, in which both shifts are negative, i.e. $X<0, \quad X_{\tau}<0 \quad$ (Fig. 8a); gear MRT-N $-x^{-} x_{\tau}^{+}$, when $X<0, X_{\tau}>0$ (Fig. 8b); gear MRT-N $-x^{+} x_{\tau}^{-}$, in which $X>0, X_{\tau}<0$ (Fig. 8c).

\section{Main variations of involute meshing}

As it has already been clarified, every gear may be zero $\left(x=0, x_{\tau}=0\right)$ or modified in radial $(x \neq 0)$ or tangential $\left(x_{\tau} \neq 0\right)$ direction, depending on the values of the shift coefficients $x$ and $x_{\tau}$. The gear drives by themselves are also grouped in different variations according to the set values of the shift coefficients $x_{1}, x_{\tau 1}, x_{2}$ and $x_{\tau 2}$ of the gears in the involute meshing.

A distinguishing feature of the main variations of the generalized involute meshing (Fig. 9) is the comparison of the reference center distance $a=m\left(z_{1}+z_{2}\right) / 2$ and the real center distance $a_{w}$, defined by equation [15]

$$
a_{w}=a \frac{\cos \alpha}{\cos \alpha_{w}}=a \frac{\cos \alpha^{\wedge}}{\cos \alpha_{w}^{\hat{w}}},
$$

where the pressure angles $\left(\alpha_{w}, \alpha_{w}\right)$ and the rack-cutter profile angles $\left(\alpha, \alpha^{\wedge}\right)$ may have different values for active and passive teeth profiles, respectively.

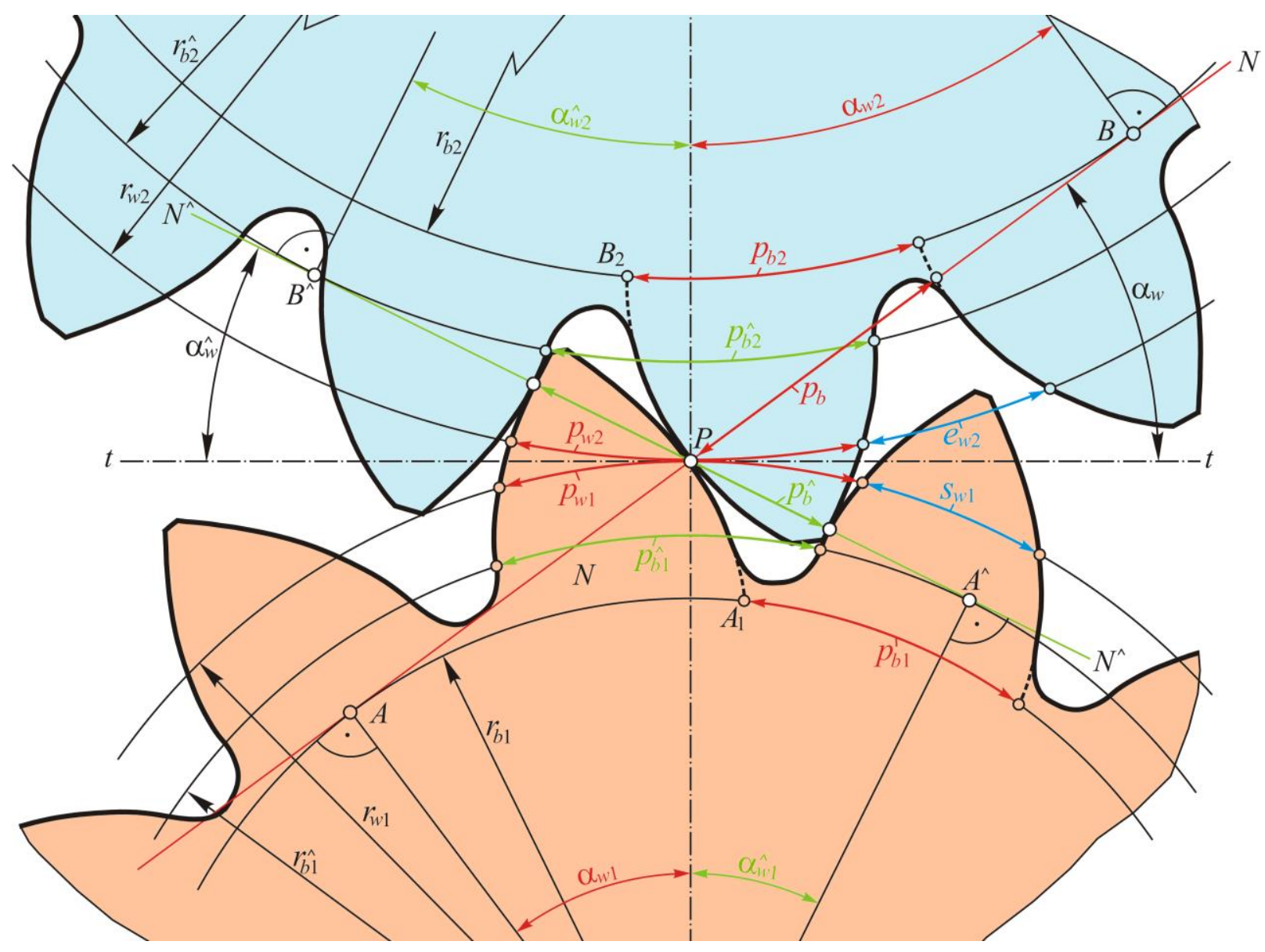

Fig. 9. Generalized involute meshing. 
From the main equation of generalized involute meshing [17], written in the form

$$
\begin{gathered}
x_{\tau \Sigma}+2 x_{\Sigma}\left(\tan \alpha+\tan \alpha^{\wedge}\right)= \\
=\left(z_{1}+z_{2}\right)\left(\operatorname{inv} \alpha_{w}+\operatorname{inv} \alpha_{w}^{\wedge}-\operatorname{inv} \alpha-\operatorname{inv} \alpha^{\wedge}\right),
\end{gathered}
$$

when

$$
x_{\Sigma}=\left(x_{1}+x_{2}\right), \quad x_{\tau \Sigma}=\left(x_{\tau 1}+x_{\tau 2}\right),
$$

it is clear that depending on the values of the angles $\alpha_{w}$, $\alpha_{\hat{w}}^{\wedge}, \alpha$ and $\alpha^{\wedge}$, three characteristic cases are obtained, shown in Fig. 10:

case I (Fig. 10a) $\Rightarrow x_{\tau \Sigma}+2 x_{\Sigma}\left(\tan \alpha+\tan \alpha^{\wedge}\right)=0$, in which $\alpha_{w}=\alpha$ and $\alpha_{w}^{\wedge}=\alpha^{\wedge}$;

case II (Fig. 10b) $\Rightarrow x_{\tau \Sigma}+2 x_{\Sigma}\left(\tan \alpha+\tan \alpha^{\wedge}\right)>0$, in which $\alpha_{w}>\alpha$ and $\alpha_{w}>\alpha^{\wedge}$;

case III (Fig. 10c) $\Rightarrow x_{\tau \Sigma}+2 x_{\Sigma}\left(\tan \alpha+\tan \alpha^{\wedge}\right)<0$, in which $\alpha_{w}<\alpha$ and $\alpha_{w}<\alpha^{\wedge}$.

From equation (6) it is clear that in the first case the center distance $a_{w}$ is equal to reference one $a$, in the second case $a_{w}>a$ and in the third one $a_{w}<a$. It is accepted the gear meshing in the second case to be called positive meshing and in the third - negative meshing.

The solving of the characteristic equation of the first case

$$
x_{\tau \Sigma}+2 x_{\Sigma}\left(\tan \alpha+\tan \alpha^{\wedge}\right)=0 \text {, }
$$

may be done in three different ways, which define three varieties of involute meshings. Therefore case $I$ has three variations:

variation Ia - zero meshing $\left(a_{w}=a\right)$, when

$$
x_{1}=x_{2}=x_{\tau 1}=x_{\tau 2}=0 ;
$$

variation Ib - equally modified meshing $\left(a_{w}=a\right)$, when $x_{\Sigma}=\left(x_{1}+x_{2}\right)=0$ and

$$
x_{\tau \Sigma}=\left(x_{\tau 1}+x_{\tau 2}\right)=0 \text {; }
$$

variation $I_{c}-$ balance-modified meshing $\left(a_{w}=a\right)$, when $x_{\Sigma}=\left(x_{1}+x_{2}\right) \neq 0$ and

$$
x_{\tau \Sigma}=\left(x_{\tau 1}+x_{\tau 2}\right) \neq 0 \text {. }
$$

Zero meshing (Fig. 10a). From the main equation of the generalized meshing it is clear that if the gear drive is zero $\left(x_{1}=0, x_{2}=0, x_{\tau 1}=0, x_{\tau 2}=0\right)$, in order to cancel the right side of the equation (7), we need inv $\alpha_{w}=\operatorname{inv} \alpha$ and inv $\alpha_{w}^{\wedge}=\operatorname{inv} \alpha^{\wedge}$. That means the active pressure angle $\alpha_{w}$ in the zero gear drive needs to be equal to the active profile angle $\alpha$ of the rack-cutter, by which both gears are cut $\left(\alpha_{w}=\alpha\right)$. Furthermore, the passive angles $\alpha_{w}^{\wedge}$ and $\alpha^{\wedge}\left(\alpha_{w}^{\wedge}=\alpha^{\wedge}\right)$ need to be equal, too. As a result of this, from the equation (6) it is clear that the center distance of the zero drive is equal to its reference center distance $\left(a_{w}=a\right)$. The zero gear drive is also characterized by coincidence between the pitch $\left(r_{w 1}, r_{w 2}\right)$ and the reference $\left(r_{1}, r_{2}\right)$ circles of both meshed gears $\left(r_{w 1}=r_{1}, r_{w 2}=r_{2}\right)$.

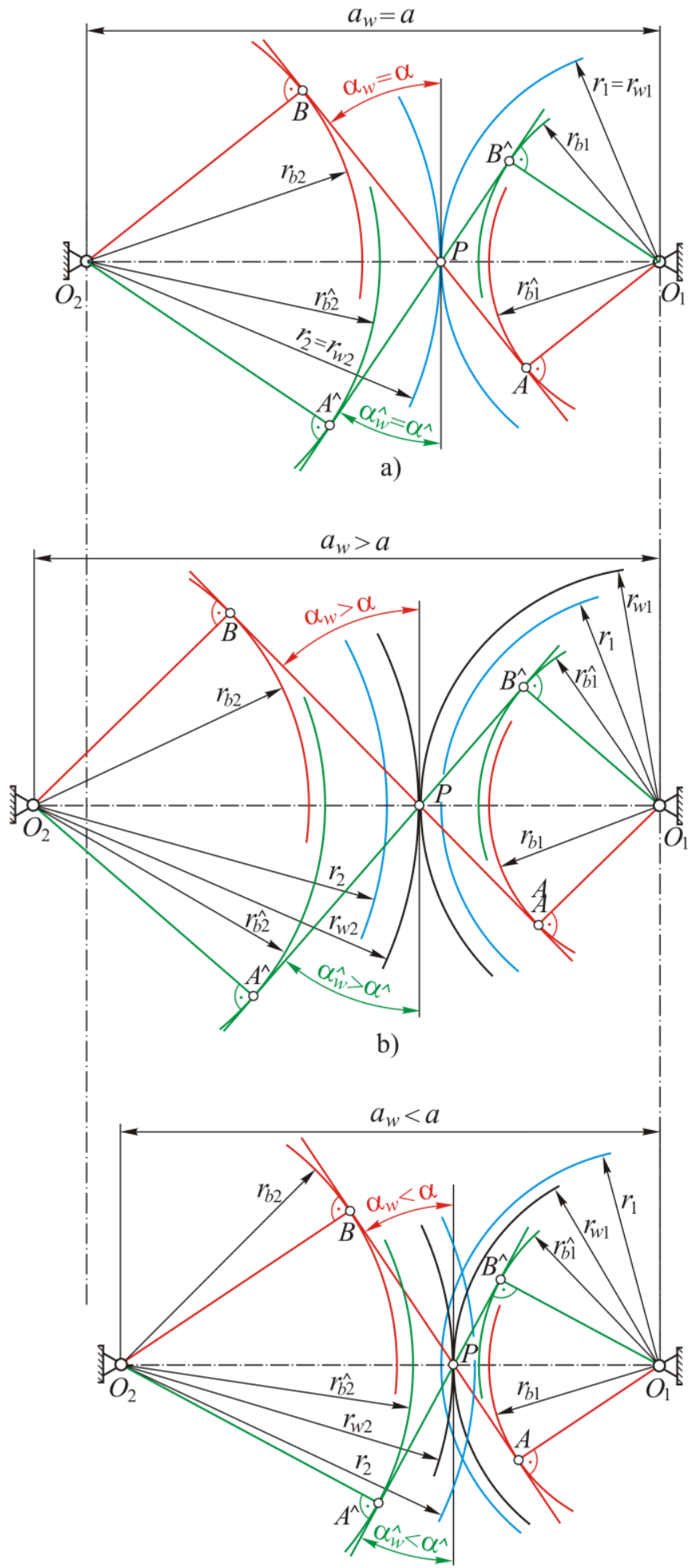

c)

Fig. 10. Types of involute meshing a) zero, balance-modified and equally-modified ; b) positive; c) negative.

Equally-modified meshing (Fig. 10a). At this meshing one of the gears (usually the small one) is positively modified $\left(x_{1}>0, x_{\tau 1}>0\right)$ and the othernegatively $\quad\left(x_{2}<0, \quad x_{\tau 2}<0\right)$. Furthermore, the coefficients $x_{1}$ and $x_{2}\left(x_{\tau 1}\right.$ and $\left.x_{\tau 2}\right)$ of both gears have equal absolute values $\left(x_{1}=-x_{2}, x_{\tau 1}=-x_{\tau 2}\right)$, and as a 
result $x_{\Sigma}=x_{1}+x_{2}=0$ and $x_{\tau \Sigma}=x_{\tau 1}+x_{\tau 2}=0$. Because the summed coefficients $x_{\Sigma}$ and $x_{\tau \Sigma}$ of the equally-modified and zero gear drives are the same (equal to zero), the relative position of the meshed gear circles shown in Fig. 10a is also equal in both gear drives. Practically, it means that in the equally-modified gear drive the requirements fulfilled are the same as these which are characteristic for the zero drive. However, there is a difference between the zero and equally-modified drives (resulting from the modification of the gears) which expresses itself in the geometric form of the teeth and their position against the meshing pole $P$. If equallymodified gear drive with bigger ratio is used instead of zero drive, it is possible at $x_{1}>0$ and $x_{\tau 1}>0$ the teeth strength (small gear teeth) and the load capacity of the gear drive to be increased.

Balance-modified meshing (Fig. 10a). When both gears are modified with different values of the coefficients $x_{1}, x_{2}, x_{\tau 1}$ and $x_{\tau 2}$, but equation (9) is fulfilled, the gear meshing is called balance-modified. The balance-modified meshing is typical only for the generalized theory proposed by the author. In this meshing the equations (10) are fulfilled.

$$
\alpha_{w}=\alpha, \quad \alpha_{w}=\alpha^{\wedge} \quad a_{w}=a, \quad r_{w 1}=r_{1}, \quad r_{w 2}=r_{2},
$$

They are characteristic for zero and equally-modified meshing, too. In this equations $r_{1}$ and $r_{2}$ are reference circles radiuses and $r_{w 1}$ and $r_{w 2}$ are pitch circles radiuses of the gears.

Using the new balance-modified meshing increases significantly the possibilities for geometric design by multiple choice for values of the shift coefficients $x_{1}$, $x_{2}, x_{\tau 1}$ and $x_{\tau 2}$.

Positive meshing (Fig. 10b). The distinguishing features of the positive meshing according to the definition written above and the equation $x_{\tau \Sigma}+2 x_{\Sigma}\left(\tan \alpha+\tan \alpha^{\wedge}\right)>0$ are:

$$
\alpha_{w}>\alpha, \quad \alpha_{w}>\alpha^{\wedge} \quad a_{w}>a, \quad r_{w 1}>r_{1}, \quad r_{w 2}>r_{2},
$$

The requirement above may be proved in another way in which by increasing the center distance $a_{w}$ of the zero drive (Fig. 10a), the positive drive shown in Fig. $10 \mathrm{~b}$ is obtained. As a result of the growth of $a_{w}$, the side gap of the meshing is increased. It can be compensated by increasing the coefficients $x_{\Sigma}$ and $x_{\tau \Sigma}$ (by increasing $x_{\Sigma}$ and $x_{\tau \Sigma}$, the teeth thickness is growing). After increasing the center distance $\left(a_{w}>a\right)$ the base circles $r_{b 1}$ and $r_{b 2}$ are placed at a greater distance and their common tangential line is also placed at a different position. Thus the pressure angle $\alpha_{w}$ increases $\left(\alpha_{w}>\alpha\right)$. At the same time, the position of the meshing pole $P$ changes which leads to increasing the radiuses of the pitch circles $r_{w 1}$ and $r_{w 2}$ (the radiuses of the reference circles $n_{1}$ and $r_{2}$ do not change) i.e. $r_{w 1}>r_{1}$ and $r_{w 2}>r_{2}$. Typical for the positive meshing is that the reference circles do not touch or cross each other.

Negative meshing (Fig. 10c). The distinguishing features of the negative meshing are formed by equations:

$\alpha_{w}<\alpha, \quad \alpha_{w}<\alpha^{\wedge} \quad a_{w}<a, \quad r_{w 1}<r_{1}, \quad r_{w 2}<r_{2}$,

which are received from (11) by substitution of the symbols $>$ and $<$. Inequalities typical for the negative gear drive (12) are clarified similarly with the reasoning above related to positive gear drive. What is different here is that the negative drive (Fig. 10c) is received from the zero drive after decreasing its center distance. As a result, the pressure angle $\left(\alpha_{w}<\alpha\right)$ is also decreased and the reference circles of the gears are crossed because $r_{w 1}>r_{1}$ and $r_{w 2}>r_{2}$.

\section{Classification of gear drives}

In Fig. 11 Depending on the different modifications of included gears, a generalized classification of involute gear drives is proposed in Fig. 11.

In this classification as well in the traditional theory, the involute gear drives in general are grouped as zero and modified. The zero gear drives are marked with $\mathbf{Z 0 0}$ and the first symbol in the mark of the modified gear drives is M (from „modification”). In the generalized classification, a gear drive is zero when the shift coefficients of both gears have no values i.e. $x_{1}=x_{2}=0$ and $x_{\tau 1}=x_{\tau 2}=0$. When one of these coefficients is different from zero, the gear drive is modified.

The modified gear drives are also divided into:

1) Equally-modified gear drives - ME (Modified Equally), in which the real and reference center distances are equal $\left(a_{w}=a\right)$, and the equations $x_{1}+x_{2}=0$ and $x_{\tau 1}+x_{\tau 2}=0$ are valid for the shift coefficients;

2) Balance-modified gear drives - MB (Modified Balance), when the real and the reference center distances are also equal $\left(a_{w}=a\right)$ due to the fact that the coefficients $x_{1}, x_{2}, x_{\tau 1}$ and $x_{\tau 2}$ fulfill the equation $x_{\tau 1}+x_{\tau 2}+2\left(x_{1}+x_{2}\right)\left(\tan \alpha+\tan \alpha^{\wedge}\right)=0$;

3) Positive gear drives- MP (Modified Positive), in which due to the fact that inequation $x_{\tau 1}+x_{\tau 2}+2\left(x_{1}+x_{2}\right)\left(\tan \alpha+\tan \alpha^{\wedge}\right)>0$ is fulfilled by the set shift coefficients, the real center distance is greater than the reference one $\left(a_{w}>a\right)$;

4) Negative gear drives - MN (Modified Negative), where $x_{\tau 1}+x_{\tau 2}+2\left(x_{1}+x_{2}\right)\left(\tan \alpha+\tan \alpha^{\wedge}\right)<0$, as a result of which the real center distance is smaller than the reference one $\left(a_{w}<a\right)$.

Depending on the values of the radial and tangential shift coefficients, the equally-modified gear drives are three types: 


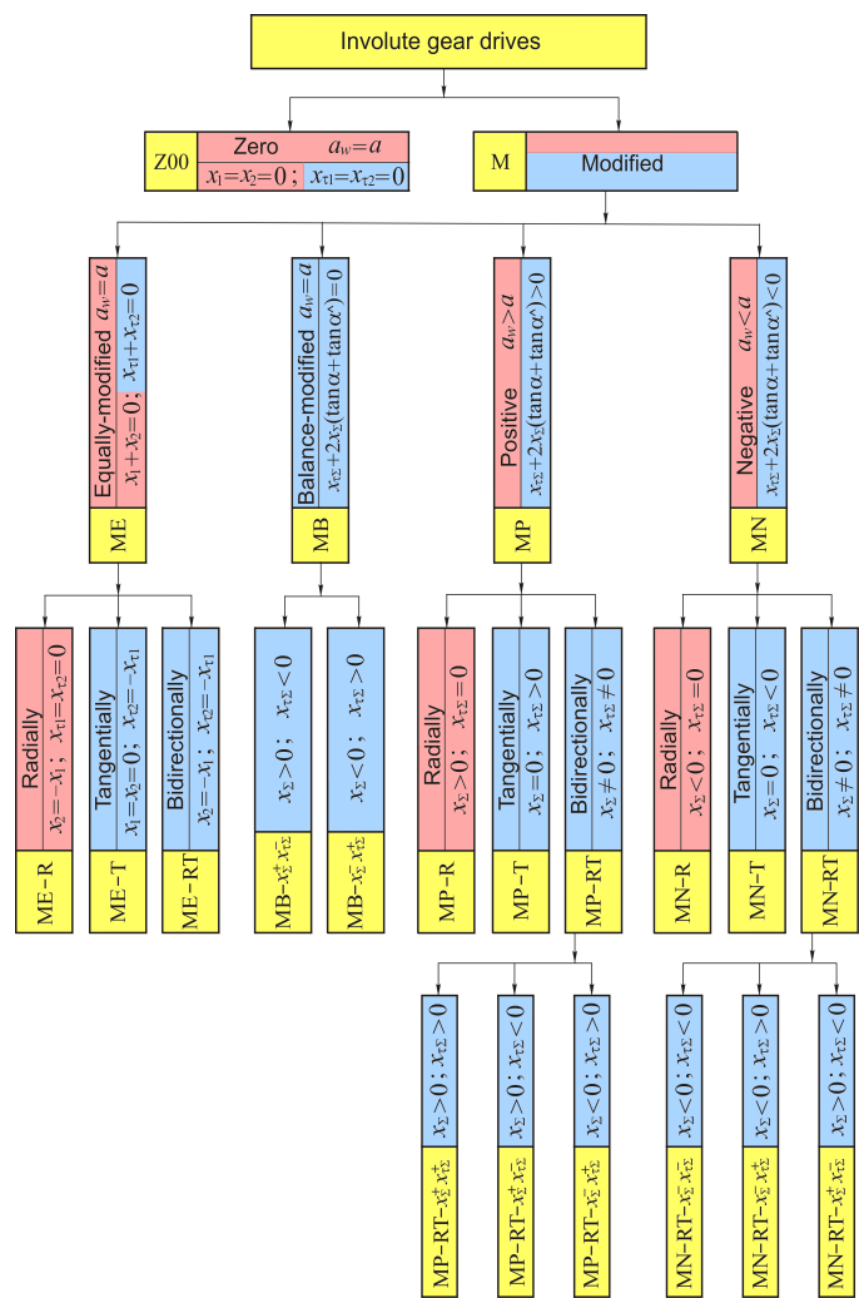

Fig. 11. Classification of involute gear drives.

1a) Radial - ME - R ( $\mathbf{R}$ - Radially), in which both gears are only radially modified and the equations $x_{2}=-x_{1}$ and $x_{\tau 1}=x_{\tau 2}=0$ are fulfilled;

$1_{b}$ ) Tangential - ME - T (T - Tangentially), in which both gears are only tangentially modified and the equations $x_{1}=x_{2}=0$ and $x_{\tau 2}=-x_{\tau 1}$ are fulfilled;

1 ) Bidirectional- ME - RT (RT - Radially and Tangentially), in which the gears are radially and tangentially modified at the same time and the equations $x_{2}=-x_{1}$ and $x_{\tau 2}=-x_{\tau 1}$ are fulfilled.

Depending on the signs of the summed coefficients $x_{\Sigma}$ and $x_{\tau \Sigma}$ of radial and tangential shift, the balancemodified gear drives are two types:

2a) MB $-\boldsymbol{x}_{\Sigma}{ }^{+} \boldsymbol{x}_{\tau \Sigma}{ }^{-}$, where the summed radial shift is positive and the summed tangential shift - negative, i.e. $x_{\Sigma}>0$ and $x_{\tau \Sigma}<0$;

2 ) $\mathbf{M B}-\boldsymbol{x}_{\boldsymbol{\Sigma}}{ }^{-} \boldsymbol{x}_{\tau \Sigma}{ }^{+}$, where the summed radial shift is negative and the summed tangential shift - positive, i.e. $x_{\Sigma}<0$ and $x_{\tau \Sigma}>0$.

Depending on the modification of the gears, the positive gear drives are divided into: $3_{\mathrm{a}}$ ) Radial $-\mathbf{M P}-\mathbf{R}$, in which the summed radial shift is positive and the summed tangential shift - zero, i.e. $x_{\Sigma}>0$ and $x_{\tau \Sigma}=0$;

$3_{\mathrm{b}}$ ) Tangential - MP - T, in which the summed radial shift is zero and the summed tangential shift is positive, i.e. $x_{\Sigma}=0$ and $x_{\tau \Sigma}>0$;

$3_{\mathrm{c}}$ ) Bidirectional - MP - RT, in which according to the signs of the summed shift coefficients $x_{\Sigma}$ and $x_{\tau \Sigma}$ three variations are possible:

$$
\begin{aligned}
& \text { MP - RT }-\boldsymbol{x}_{\Sigma}{ }^{+} \boldsymbol{x}_{\tau \Sigma}{ }^{+} \text {, when } x_{\Sigma}>0 \text { and } x_{\tau \Sigma}>0 \\
& \text { MP - RT }-\boldsymbol{x}_{\Sigma}{ }^{+} \boldsymbol{x}_{\tau \Sigma} \text {, when } x_{\Sigma}>0 \text { and } x_{\tau \Sigma}<0 ; \\
& \text { MP - RT }-\boldsymbol{x}_{\Sigma}{ }^{-} \boldsymbol{x}_{\tau \Sigma}{ }^{+} \text {, when } x_{\Sigma}<0 \text { and } x_{\tau \Sigma}>0
\end{aligned}
$$

The negative gear drives according to the modification of the gears are also divided into three types:

3a) Radial - MN - R , in which $x_{\Sigma}<0$ and $x_{\tau \Sigma}=0$;

$3_{\mathrm{b}}$ ) Tangential - MN-T, in which $x_{\Sigma}=0$ and $x_{\tau \Sigma}<0$;

3c) Bidirectional - MN - RT, in which according to the signs of the summed shift coefficients $x_{\Sigma}$ and $x_{\tau \Sigma}$ three variations are possible:

MN - RT $-\boldsymbol{x}_{\Sigma}{ }^{-} \boldsymbol{x}_{\tau \Sigma}{ }^{-}$, when $x_{\Sigma}<0$ and $x_{\tau \Sigma}<0$;

MN - RT $-\boldsymbol{x}_{\Sigma}{ }^{-} \boldsymbol{x}_{\tau \Sigma}{ }^{+}$, when $x_{\Sigma}<0$ and $x_{\tau \Sigma}>0$;

MN - RT $-\boldsymbol{x}_{\Sigma}{ }^{+} \boldsymbol{x}_{\tau \Sigma}{ }^{-}$, when $x_{\Sigma}>0$ and $x_{\tau \Sigma}<0$.

\section{Conclusions}

- In the GT proposed the methodology related to gears modification and the modification of the involute meshing is developed further. In this regard, along with traditional radial shift of the basic rack realized by the rack-cutter, a tangential shift of the side profiles of these racks is introduced. The capabilities of the geometric design are significantly increased due to the bidirectional modification.

- From the classification proposed in Fig. 2, it is clear that the total number of the different types of involute gears is thirteen (one unmodified and twelve modified differently). For comparison, in the traditional classification the gears are three types - one zero and two radially modified gears, respectively with positive and negative signs. The existence of 10 more varieties in the classification proposed is a condition for developing the opportunities of the involute meshing in GT.

- The total number of the different types of involute gear drives in GT (Fig. 11) depending on the modification method is sixteen - one zero, three equallymodified, two balance-modified, five positive and five negative. For comparison, in the TT the gear drives are four types - one zero, one balance-modified, one positive and one negative. This addition of 12 new variations in the classification proposed considerably extends the area of existence of the involute meshing. 


\section{References}

1. J.R. Colbourne, The Geometry of Involute Gears (Berlin: Springer-Verlag, p. 532, 1987)

2. F.L. Litvin, A. Fuentes, Gear geometry and applied theory (Cambridge University Press, Cambridge, 2004)

3. N. Minchev, V. Jivkov, P. Stoianov, O. Alipiev, Theory of mechanisms and machines (Softtreide, p. 595, in Bulgarian, 2018)

4. P. Nenov, B. Kaloyanov, E. Angelova, Geometrical blocking contours as an instrument for hightechnology design of geometry of involute external and internal gear drives, Mechanisms and Machine Science. Power Transmissions 379-388, (2013)

5. E.B. Vulgakov, Theory of Involute Gears (Mashinostroyenie, Moscow, in Russian,1995)

6. A. Kapelevich, Direct Gear Design (CRC Press, Taylor \& Francis Group, p. 324, 2013)

7. A.L. Kapelevich, Geometry and design of involute spur gears with asymmetric teeth. Mech. Mach. Theory, 35, 117-130 (2000)

8. F. Karpat, O. Dogan, C. Yuce, S. Ekwaro-Osire, An improved numerical me-thod for the mesh stiffness calculation of spur gears with asymmetric teeth on dynamic load analysis. Advances in Mechanical Engineering, 9 (8), 1-12, (2017)

9. Fetvaci, C., Imrak, C. Mathematical model of a spur gear with asymmetric involute teeth and its cutting simulation. Mechanics Based Design of Structures and Machines, 36, 34-46, (2008)

10. P. Marimuthu, G. Muthuveerappan, Investigation of load carrying capacity of asymmetric high contact ratio spur gear based on load sharing using direct gear design approach. Mech. Mach. Theory, 96 (1), 52-74 (2000)
11. R.P. Sekar, Determination of load dependent gear loss factor on asymmetric spur gear. Mech. Mach. Theory, 135 , 322-335, (2019)

12. A. Kapelevich, Asymmetric Gearing (CRC Press, p. 287, 2018)

13. V. Starzhinsky, N. Ishin, A. Goman, Perfection of quality parameters of plastic gears by using asymmetric tooth profile. Machine Design $3(2)$, 109-114, (2016)

14. O. Alipiev, Generalized model of involute meshing, Mechanics of Machines. 116, 11-18, (in Bulgarian, 2016)

15. O. Alipiev, Geometric calculation of involute spur gears defined with generalized basic rack. Theory of Mechanisms and Machines, 12 (2) 60-70, (in Russian, 2008)

16. O. Alipiev, Generalized determination of the contact ratio at involute tooth system, Mechanics of Machines, 3, 63-70 , (in Bulgarian , 2008)

17. O. Alipiev, S. Antonov, Basic equation and its application in the generalized geometric theory when projecting the involute gearings with external engagement. Mechanics of Machines 68, 75-79, (in Bulgarian, 2007)

18. O. Alipiev, S. Antonov, T. Grozeva, Generalized model of undercutting of involute spur gears generated by rack-cutters. Mech. Mach. Theory 64, 39-52, (2013)

19. O. Alipiev, Generalized basic rack at involute gears, Mechanics of Machines. 116, 19-24, (in Bulgarian, 2016)

20. O. Alipiev, Geometric design of involute spur gear drives with symmetric and asymmetric teeth using the Realized Potential Method, Mech. Mach. Theory, 46, 10-32, (2011)

21. O. Alipiev, Generalized theory of the involute gear meshing (Sc. D. Thesis, University of Ruse, Ruse, p. 447, in Bulgarian, 2017) 